\title{
ATTACK PATTERN ANALYSIS FRAMEWORK FOR MULTIAGENT INTRUSION DETECTION SYSTEM
}

\author{
GRZEGORZ KOLACZEK \\ Institute of Information Science and Engineering, \\ Wroctaw University of Technology, \\ Wybrzeże Wyspiańskiego 27, 50-370 Wrocław, Poland \\ E-mail: grzegorz.kolaczek@pwr.wroc.pl \\ KRZYSZTOF JUSZCZYSZYN \\ Institute of Information Science and Engineering, \\ Wroctaw University of Technology, \\ Wybrzeże Wyspiańskiego 27, 50-370 Wrocław, Poland \\ E-mail: krzysztof.juszczyszyn@pwr.wroc.pl \\ Received: 16-01-2008 \\ Revised: $\quad 30-06-2008$
}

\begin{abstract}
The paper proposes the use of attack pattern ontology and formal framework for network traffic anomalies detection within a distributed multiagent Intrusion Detection System architecture. Our framework assumes ontology-based attack definition and distributed processing scheme with exchange of communicates between agents. The role of traffic anomalies detection was presented then it has been discussed how some specific values characterizing network communication can be used to detect network anomalies caused by security incidents (worm attack, virus spreading). Finally, it has been defined how to use the proposed techniques in distributed IDS using attack pattern ontology.
\end{abstract}

Keywords: ontology, intrusion detection, agent systems, traffic analysis.

\section{Introduction}

In order to process intrinsically distributed information, most of modern IDS systems are organized in a hierarchical architecture ${ }^{4}$, consisting of low level nodes which collect information and management nodes which aim to detect large-scale phenomena. The task of management nodes is to reduce the amount of the data processed, identify attack situations as well as make decisions about responses ${ }^{10}$.

In our approach it is assumed that the network system consists of the set of nodes. There are also two types of agents in our multiagent system: monitoring agents (MoA) and managing agents (MA) $)^{7,8,9}$. Monitoring agents observe the nodes, process captured information and draw conclusions that are necessary to evaluate the current state of system security within their areas of responsibility. Managing agents are responsible for gathering information from MoA agents and generating reports about global threats and ongoing attacks. Each agent MoA monitors its own area of responsibility consisting of the set of network nodes. It is commonly known that in the case of worm attack there occur at least two kinds of anomalies: in observed traffic characteristics and in communication scheme which tends to be constant under normal conditions. In this context the system properties observed by the agent MoA in the proposed architecture will fall into two basic (and physically different) categories: traffic measurement, communication pattern measurement. The attack recognition is being made on the basis of them.

The MoA agent's algorithm for decision making process is invoked periodically and uses observed values as input data. MoA also stores acquired values thus creating the history of system behavior. 
Our approach assumes the use of attack pattern ontology which allows to define known attack schemes. Typical attack definition consists of a combination of attack symptoms which are abnormal values of the network variables being observed by the MoAs. The information about attack symptoms is exchanged between the agents in the form of communicates which associate an attack probability value with each variable. The entire framework is extensible - the attack pattern ontology and the set of observed variables may be easily extended in order to incorporate new concepts and variables needed to define new attack types.

The rest of the paper is structured as follows. After short introduction (sec. 2) we present a basic set of network variables which are observed by the agents (sec. 3). The statistics used to determine the probability of abnormal values of any given variable are defined in sec. 4. Also, the concept of observation of network traffic structure in order to reason about abnormal situations is presented in sec. 5 along with appropriate variables. Sec. 6 introduces attack pattern ontology which is used to define exemplary attack in sec. 7. Sec. 8 explains the reasoning procedure for attack detection.

\section{Network Traffic Anomalies and Intrusion Detection}

Intrusion detection systems (IDS) have been proposed as an approach to cope with current security problems. The aim of the intrusion detection is discovering of all abnormal states of the system in relation to the network traffic, users activity and system configuration that may indicate violation of security policy ${ }^{1,2}$. But although the IDS idea is very simple, implementation of such systems has to deal with a lot of practical and theoretical problems. Difficulties with building intrusion detection systems arise from a complexity of the structure of attacks symptoms, distributed nature of the network systems and dynamics of the source of threats especially the problems of encoding new intrusions scenarios. The security assessment of a network system requires application of complex and flexible mechanisms for monitoring values of system attributes that have an influence on the security level of all network system.

Another important element is an effective computational mechanism for evaluating the states of system security on the basis of incomplete, uncertain and inconsistent resources. Finally, the algorithms of machine learning to detect new intrusions pattern scenarios and recognize new symptoms of security system breach in order to update the security system knowledge base must be defined.

\section{Evaluation of Network Traffic Anomalies}

Traffic attributes that are especially important (because their rapid change during typical attacks) and used during process of anomaly detection are ${ }^{1}$ :

- $\quad$ source and destination IP address,

- $\quad$ source and destination port,

- number of bytes and packets sent to the remote hosts,

- number of bytes packets received by the local host,

- TCP flags, especially SYN, RST and FIN flags

- duration of the connection.

The values of variables describing these attributes are collected and processed by intrusion detection system in a purpose to identify any anomalous behavior. The simplest decision mechanism applied in intrusion detection system uses threshold test to find out if the observed value is typical or it can be classified as anomalous. This preliminary observations will be then used in metadata-based detection environment in order to reason about network attacks.

\subsection{Network traffic related variablesuUsed in evaluation}

In our approach we observe: source/destination IP and port number, number of bytes sent/received and ration of number of SYN packets to FIN packets. These attributes were selected because a significant number of security incidents like denial of service attacks (DoS and Distributeid DoS), worm attacks, scanning cause changes in their values and so it could be recognized as an anomalous state. For example intrinsic nature of DoS/DDoS or intrusive system scan attacks makes that existing in the normal state of the system communication patterns must be effected by these events $^{8}$. Communication patterns are related to the attributes like IP address of source/destination host or port number of the required network service. Similarly, other attacks like worm, alpha or flash crowd will also have an effect on different traffic related attributes like average duration of the connection or average number of bytes sent by a host ${ }^{12}$.

Raw data obtained as a result of above mentioned network traffic parameters observation must be 
transformed to get some useful information that can be used to identify the deviation between the current system's state and another state that is supposed to characterize the normal system behavior. In the following sections we describe our approach to transformation of traffic related attributes values.

\subsection{Source/destination IP address and port number}

To measure changes in IP address and port number space we will observe a value of Shannon entropy related to these attributes ${ }^{13}$. Entropy values are calculated for separate time periods. The length of the period can be a subject of more detailed discussion ${ }^{1}$, however we assume that it is possible that different monitoring agents (MoA) use various periods length.

This means that we will evaluate, collect and investigate the following network variables:

- S_IP (ti) - entropy of source IP address in the period $t_{i}$,

- D_IP(ti) - entropy of destination IP address in the period $t_{i}$,

- D_Port(ti) - entropy of destination port number in the period $t_{i}$,

- S_Port(ti) - entropy of source port number in the period $t_{i}$.

Entropy value is evaluated from standard formula:

$$
e=-\sum_{i=0}^{N} p_{i} \log p_{i}, \quad p_{i}=\frac{n_{i}}{\sum_{i=0}^{N} n_{i}}, 0 \leq i \leq N,
$$

where:

$N \quad-$ cardinal number of IP address/port number set,

$n_{i} \quad-\quad$ number of packets with a particular source/destination IP address/port number observed in the period $\mathrm{t}_{\mathrm{i}}$,

$\sum_{i=0}^{N} n_{i}-\quad \begin{aligned} & \text { total number of packets observed in the } \\ & \text { period } t_{\mathrm{i}}\end{aligned}$

As for some $\mathrm{t}_{\mathrm{i}}$, the value of $\sum_{i=0}^{N} n_{i}$ can be equal to zero (no traffic observed in $t_{i}$ period), we assume that in these periods entropy value is also zero.

Any untypical changes of variables values related to IP address or port number entropy can be treated as a sign of anomalous behavior of the monitored system. Especially we can assign some threshold value which will indicate the state of anomalous entropy level. E.g. AS_IP will be a constant describing the value of acceptable S_IP level

\subsection{Number of bytes and packets}

Changes of entropy values are strictly related to changes of communication patterns. Using this measure of traffic parameters, some sort of anomalies caused by intrusive actions like DoS or system scan can be detected. However, other types of intrusions do not have to disturb communication patterns. For example so called topological worms using internally generated target lists tries to infect only remote targets well known by the infected host. Well known, means that instead of performing random scan to find vulnerable hosts, the worm tries to discover the local communication topology and infect only hosts which sent or received data to or form infected host ${ }^{14}$.

Like it has been shown in section 3.2 the values describing number of bytes and packets exchanged by a host will be obtained as a result of observation of incoming and outgoing traffic in each of constant size period while it is observed by MoA.

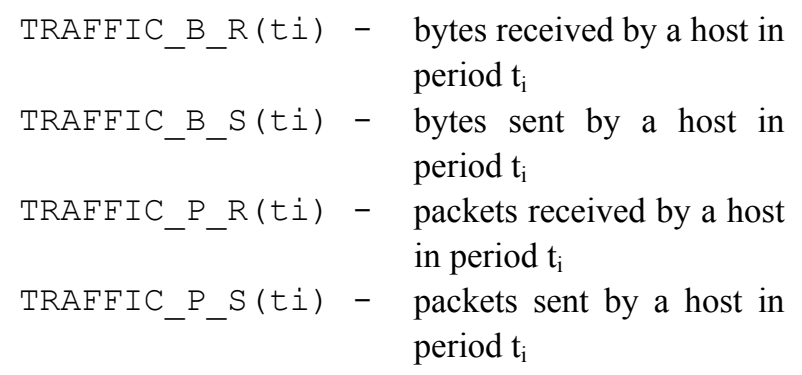

Also a traffic threshold value can be assigned and described by e.g. ATRAFFI_B_R, ATRAFFI_B_S, etc.

\subsection{TCP flags}

The TCP flags are important source of information about host's connections state. Typical TCP connection have three phases: connection establishment, data transfer, connection termination. Each phase uses packets with some standard sequences of TCP flags, especially TCP flags brings information about current connection state. However, this information may be 
incorrect while an intruder can manipulate the packet's content to reach some particular aim (e.g. the intruder tries to obtain information about services activated by host by performing system scan or simmilar effect can be observed during DoS/DDoS attacks ${ }^{10}$.

In our approach we measure a difference between number of SYN packets sent and RST and FIN packets received.

$$
\mathrm{TCP} \_\mathrm{FLAG}=p_{t_{i}}^{s y n}-p_{t_{i}}^{r s t}-p_{t_{i}}^{\text {fin }}
$$

where:

\begin{tabular}{|c|c|}
\hline _RLAU & $\begin{array}{l}\text { - parameter indicating tempor } \\
\text { start/end connection ratio }\end{array}$ \\
\hline$p_{t}$ & $\begin{array}{l}\text { - number of sent TCP packets with } \\
\text { SYN flag set, }\end{array}$ \\
\hline$p^{\prime}$ & $\begin{array}{l}\text { - number of received TCP packets } \\
\text { with RST flag set, }\end{array}$ \\
\hline fin & $\begin{array}{l}\text { - } \text { number of received TCP packets } \\
\text { with FIN flag set }\end{array}$ \\
\hline
\end{tabular}

In normal conditions, in long time observation we should get the mean value of TCP_FLAG near zero. Intrusive actions like system scanning, DoS attacks, may cause the temporal distortion of the mean value of TCP_FLAG.

\subsection{Duration of the connection}

Duration of a connection is another characteristic attribute in anomaly detection process ${ }^{1}$. During various types of attacks, this value will be affected and so an anomaly may be detected. For example worm infection will generate a large number of connections with similar duration. This worm related connections should change also the observed mean values of connection duration that has been observed in a system. We evaluate simple mean value of connections' duration that have been observed in period $t_{\mathrm{i}}$.

$c_{t_{i}}$ - mean value of duration of connections that have been observed in a period $t_{i}$

\section{Traffic Statistics}

In section 3 a few traffic related variables have been presented. Values of these variables can be used to obtain useful information about system security incidents. Apart from collecting these values, intrusion detection mechanism must preprocess them to reduce the probability of misinterpretation and so called falsepositive alarm.

Our approach uses Mark Burgess (MB) technique to find out anomalous behavior. This technique of anomaly detection has been described $\mathrm{in}^{2,3}$. The main assumptions made in his framework are as follows.

MB defines iterative expectation function. Let $\mathrm{q}$ be an observation, and $\left.\left\langle<\mathrm{q}_{\mathrm{i}}\right\rangle\right\rangle$ be the i-th estimator of the average, with geometric fall-off, then $\left\langle<\mathrm{q}_{\mathrm{i}}>>\right.$ may be defined by the recurrence relation:

$$
<<\mathrm{q}>>_{\mathrm{i}+1}=\left(\mathrm{q} \mid<<\mathrm{q}>>_{\mathrm{i}}\right),<<\mathrm{q}>>_{0}=0
$$

where

$$
\left(q_{1} \mid q_{2}\right)=\frac{w q_{1}+\bar{w} q_{2}}{w+\bar{w}}, w, \bar{w}-\text { const }
$$

The other fundamental notion for MB analysis is pseudo-periodic function:

$$
\begin{gathered}
q(t)=\sum_{n=0}^{\infty} q(n P+\tau) \equiv \sum_{n=0}^{\infty} \chi_{n}(\tau), \\
\text { where } 0 \leq \tau<P
\end{gathered}
$$

Such pseudo-periodic function can be characterized by two kinds of average: average over corresponding times in different periods (topological average $<\chi(\tau)>_{T}$ ), and average of neighhoring times in a single period (local average $\left\langle\chi(\tau)>_{P}\right)^{19,20}$. Limited memory versions of these deviations are given by the following formulas:

$$
\begin{gathered}
\sigma_{<<T>>}(\tau) \equiv \sqrt{\left.<<\left(\delta_{<<T>>} \chi\right)^{2}\right\rangle_{T}} \\
\sigma_{<<P>>}(n) \equiv \sqrt{<<\left(\delta_{<<P>>} \chi\right)^{2}>>_{P}}
\end{gathered}
$$

where, for any measure $\mathrm{X}$ :

$$
\begin{gathered}
\left(\delta_{<<P>} X\right) \equiv X-<<X>>_{P} \\
\left(\delta_{<<T>} X\right) \equiv X-<<X>>_{T}
\end{gathered}
$$

These averages are calculated by replacing the evenly weighted sum over the entire history by an iteratively weighted sum that falls off with geometric degradation. The additional positive consequence of this definition is that in order to obtain all information, one 
only needs to retain and update the mean and the variance.

In contemporary network, traffic congestion is avoided by packet switching. The traffic has been isolated to 'parallel' branches of a network spanning tree. Network nodes or hosts occupy points at the leaves of these branches and therefore experience an individual (subjective) view of the network traffic. The concept of an anomaly is also a very subjective one because what is unusual for one node is a regular occurrence for another. One of the best places in the network where incidents may be tracked down and so anomalies may be reveal are the network nodes.

As stated above, anomalousness is a subjective judgment, made within the context of past experience, and can be codified into a 'policy' about what is sufficiently anomalous to warrant a response. So, we look for a potential anomalous behavior by comparing current observation to learned experience. If the event looks probable, we can consider it as the evidence derived from a supporting semantic model. As in our approach a Monitoring Agent is responsible for interpretation of the data stream arriving to the particular node, an overall situation assessment must be based on a set of communicates concerning the the traffic-related variable (measured in network nodes) coming from monitoring agents (MoA) and gathered by the managing agent (MA).

However, it is possible that some attacks may be unnoticed using such method. First thing is that MoA may misjudge some observation and as a result it doesn't report about abnormal state to the MA. This may be because of low frequency of observations, too large areas of responsibility assigned to monitoring agents (and resulting delays in communication and reports) or the characteristics of the attack itself (like for the abovementioned topological worms).

In order to deal with these difficulties an additional technique is proposed to be used on the level of Managing Agent (MA). The technique is based on observation of so-called communication patterns and allows to reason about abnormal system behaviour independently of the actual observations of network traffic variables.

\section{Communication Patterns}

Network traffic show some quantitative and topological features that appear to be invariant and characteristic for given network ${ }^{16,17}$. Moreover, general rules underlying that features are the same for almost any network of remarkable size. These distinct features concern topology of network communication, considered as origin-destination flows graph, the distribution of data volumes sent between destinations and the in/out ratio of data sent between nodes and the outside world.

With respect to these properties, wide range of network attacks can be detected by observation of communication patterns and comparison between existing under normal state of the network to new ones which occur under attack. For example, in case of Internet worm attacks, within a network there could be scanning and attack flows which differ substantially from normal network activity ${ }^{18}$.

Another invariant for a long time periods and different scales (subnet sizes) or traffic types (protocols) is proportion between a number of internal (Fan-in) and external (Fan-out) data flows ${ }^{15}$. Experiments showed that both Fan-in and Fan-out for given node and their distribution for all nodes tend to be constant under normal conditions. Under worm attack the structure of communication is heavily affected and the distribution changes. There is also a detectible dependence between worm propagation algorithm, and communication pattern disturbance ${ }^{17}$.

Monitoring agents of proposed IDS system will gather information about communication within the network. Then the existing communication patterns will be discovered. The system will be viewed as a graph consisting of nodes (each monitoring agent will have a set of nodes under control) and edges which appear if there exists data flow between given pair of nodes. In our approach we are interested in tracking the following communication patterns:

\subsection{Clustering coefficient for a given node.}

The clustering coefficient $c$ is the probability that two nearest neighbours of vertex $i$ are also neighbours of each other. The value of $c$ provides a quantitative measure for cliques in communication graph. For node $i$ clustering $c_{i}$ is given by:

$$
c_{i}=\frac{2 k_{i}}{n_{i}\left(n_{i}-1\right)}
$$

where $n_{i}$ is the number of its neighbours and $k_{i}$ - the number of connections between them. High (close to 
one) $c$ means that a node belongs to a clique in considered graph.

\subsection{Fan-in and Fan-out ratios.}

Fan-in is the number of nodes that originate data exchange with node $i$, while Fan-out is the number of hosts to which $i$ initiates conversations.

According to results listed in previous section the above patterns are invariant during most time of normal system activity or change in a predictive way. But while attack appears they will change leading to alert and taking chosen countermeasures.

The data about communication are stored in the form of $M_{c}$ matrix. The values of $M_{c}$ are set according to the following rules:

$$
M_{c}(i, j)=\left\{\begin{array}{c}
1: \text { node } i \text { communicates with node } j \\
0: \text { there is no communication between } \\
\text { nodes } i \text { and } j
\end{array}\right.
$$

Let's denote the state of $M_{c}$ in $t$ as $M_{c}^{t}$. We assume tracking three communication patterns: Fan-in (from here on denoted as $f_{i n, i}^{t}$ for node $i$ at the time moment $t$ ), Fan-out $\left(f_{\text {out }, i}^{t}\right)$ and clustering coefficient $\left(c_{i}^{t}\right)$.

Now we should investigate which values of the parameter are normal (safe). Assume that the MoA's history consists of a number of observations of Fan-in values from some starting point up to current time $t$. So we have $f_{i n, i}^{1}, f_{i n, i}^{2}, \ldots f_{i n, i}^{t}$. Let us now consider the Fan-in as a random variable $F_{i n, i}$. Thus, $\left(f_{i n, i}^{1}, f_{i n, i}^{2}\right.$, $\ldots f_{i n, i}^{t}$ ) is a sample of size $t$ of $F_{i n, i}$. We also assume all of the $f_{i n, i}$ to be independent. It is commonly known that the mean value and the variance of $F_{i n, i}$ can be estimated by the following formulae:

$$
\begin{gathered}
\overline{\mathrm{F}}_{i n, i}=\frac{1}{m} \sum_{k=1}^{t} f_{i n, i}^{k} \\
\mathrm{~S}_{\mathrm{in}, \mathrm{i}}=\frac{1}{t-1} \sum_{k=1}^{t}\left(f_{i n, i}^{k}-\bar{F}_{i n, i}\right)^{2}
\end{gathered}
$$

$\overline{\mathrm{F}}_{i n, i}$ and $\mathrm{S}_{\mathrm{in}, \mathrm{i}}$ are thus the estimations of mean value and the variance of $F_{i n, i}$. Obviously the bigger our sample is, the better they approximate $E\left(F_{i n, i}\right)$ and
$\operatorname{Var}\left(F_{i n, i}\right)$ respectively - from this point we assume that the observations' number is big enough to state that $E\left(F_{i n, i}\right)$ and $\operatorname{Var}\left(F_{i n, i}\right)$ are known.

Let also $E\left(F_{\text {out }, i}\right)$ and $\operatorname{Var}\left(F_{\text {out }, i}\right)$ for the Fan-in, as well as $E\left(C_{i}\right)$ and $\operatorname{Var}\left(C_{i}\right)$ for clustering coefficient be defined in the same way.

From the Chebyshev's inequality we can estimate the upper bound of the probability that $|\bar{F}-x|$ is greater than $k S$, where $\bar{F}$ and $S$ are mean value and the variance of $X$, while $X$ denotes the random variable related to $x$ (in this case one of the following: $\left.F_{\text {in }, i}^{t}, F_{\text {out }, i}^{t}, C_{i}^{t}\right)$.

MoA can calculate the following value:

$$
d=1-\frac{1}{\alpha k^{2}}
$$

where $\alpha$ is a coefficient which value should be set during a process of IDS tuning to the real network conditions and parameter $k$ is:

$$
k=\left\{\begin{array}{cl}
1 & \text { if }\left|\frac{\bar{F}-x}{\sqrt{S}}\right|<1 \\
\left|\frac{\bar{F}-x}{\sqrt{S}}\right| & \text { if }\left|\frac{\bar{F}-x}{\sqrt{S}}\right| \geq 1
\end{array}\right.
$$

Finally the MoA can use the $d$ parameter value to evaluate the level of Fan-in and Fan-out anomalous behaviour. The small $d$ parameter values (near 0 ) are related to typical Fan-in and Fan-out while $d$ values near 1 denote anomaly.

Note, that detection of the anomaly related to communication pattern is inferred globally by the Monitoring Agent. If, at the same time, no attack is detected on the basis of traffic analysis, the MA starts the following procedures:

1. Raises the frequency of observations of network traffic variables (actually done by the MoAs).

2. Checks the MoA's areas of responsibility; if they are too large, or there are communication delays, reduces them and creates new MoAs if necessary.

3. Generates an alert about possible critical situation.

The observation of communication patterns allows to tune the system and track anomalies which are hard to detect on the basis of traffic observations alone. 


\section{Attack Pattern Ontology}

On the level of Monitoring Agents (MoA) we postulate the following generic form of communicate about network variables:

$\mathrm{MoA}_{1}\left(\mathrm{~N}_{1}, \mathrm{~V}_{1}\right)=x$, where $x \in[0,1]$

which should be read:

„Monitoring agent $M o A_{1}$ states, that the value of network variable $V_{1}$ measured in node $N_{I}$ is normal (i.e. characteristic for the absence of attack) with probability $x "$.

It is also assumed, that for any $V_{1}$ exists some threshold value $A_{i}$, such that any value $\operatorname{MoA}_{1}\left(N_{1}, V_{1}\right)<A_{i}$ means that we experience an abnormal (suggesting that there's an attack) value of $\mathrm{V}_{1}$.

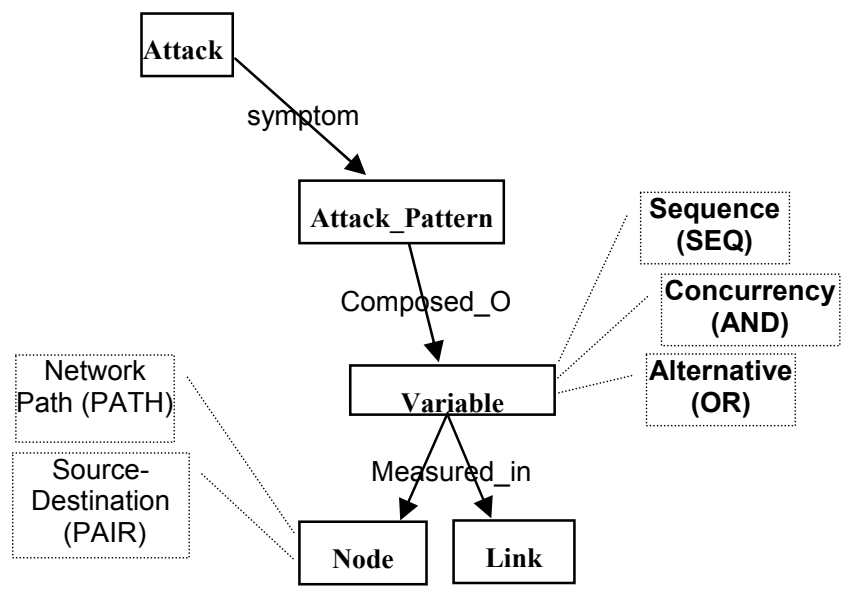

Fig. 1. Core attack pattern ontology.

Moreover, the core ontology containing basic concepts for defining attack patterns is proposed in order to help in defining attacks and to simplify network variable-based computations. The ontology contains basic concepts like Attack which is characterized by certain Attack_Pattern (Fig.1), which in turn is defined by certain set of observations of the network variables. As mentioned above, the observations are given in form of MoA's communicates about probability of anomalous variable value. Our ontology contains also specific operators which allow to define a sequence (SEQ) of communicates, their concurrency (AND) or alternative (OR). It is also possible to consider paths (i.e. sequences of nodes) in network graph (PATH) and origin-destination pairs of network nodes (PAIR).

This simple ontology will be further extended during future system development in order to incorporate more concepts representing system components and possible complex attack scenarios. Then a possibility of generating high-level communicates about system state will be evaluated.

Now we may define simple network attack, which will help us to illustrate how to use the attack pattern ontology.

\section{Attack Pattern Definition}

Let us consider so-called Reflector Attack which takes place according to the following scheme:

An attacker prepares the attack by compromising several vulnerable hosts which create a network of so called "zombie" hosts.

The attacker initiates the attack and orders all "zombie" hosts to send spoofed SYN packets with the source address set to the victim's IP address to an agent ("reflector") host.

The agent ("reflector") host responds to this SYN packet by sending a SYN|ACK or a RST packet to the source address, which is actually the victim's IP address.

The victim replies with RST packets to reflector's SYN/ACK packets and with no packet to reflector's RST packets

The "zombies" send a continual storm of theses packets, thus causing the victim host to be flooded by innocent agent host ("reflector" host).

With big number of Reflectors, the Target is down in a short time.

All this activity has obvious influence on network variables being measured. But now we may define Reflector Attack using our ontology:

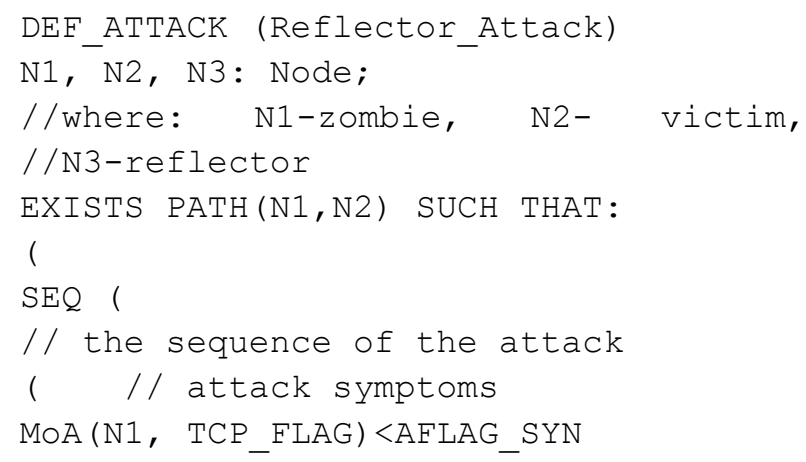




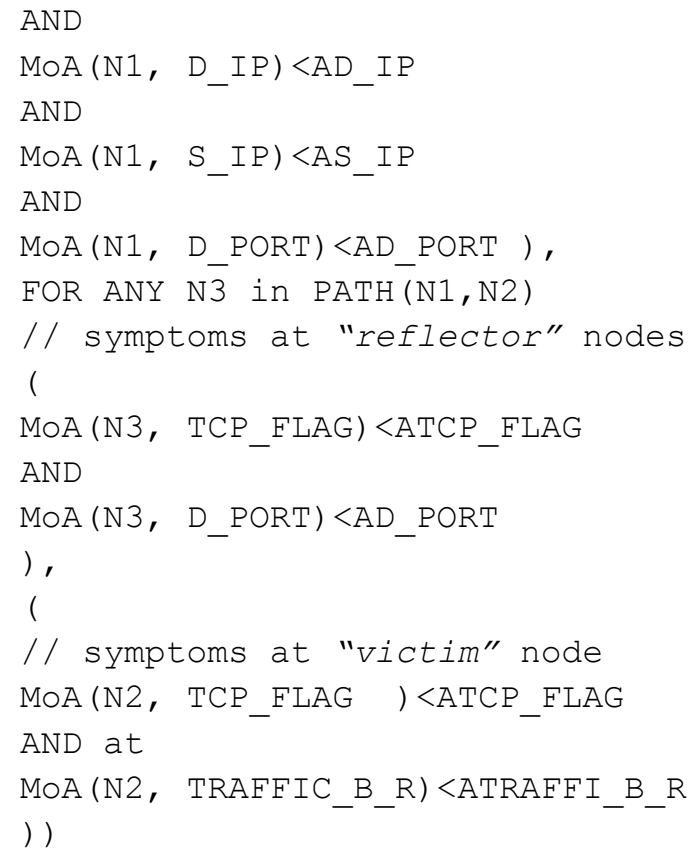

In the above definition we use earlier defined the following variables:

D_IP, S_IP

D_PORT

TRAFFIC_B_R

TCP FLAG

Note, that any single observation (like: $\mathrm{MoA}$ (N1, TCP FLAG) $<$ TCP FLAG) doesn't have to (and typically does not) imply that we are experiencing an attack. But taking them together we see that given pattern of observations clearly suggests known type of the attack.

\section{Reasoning about Attacks}

Our distributed intrusion detection system recognizes and alarms about security events according to MoA observations and MA decisions. The accurateness of final IDS decision depends on MoAs evaluation of observed values and MA ability to correctly recognize attack patterns using data delivered by MoA and attack pattern ontology.

Reasoning about attack that is performed by IDS can be described by the following procedure.

Each MoA observes and evaluates a set of variables described in sections $3.2-3.5$. During this step MoA updates variables values so they represent the current system state. Variable list obtained by $\mathrm{MoA}_{1}$ may be similar to the following example:

TCP FLAG $=143$

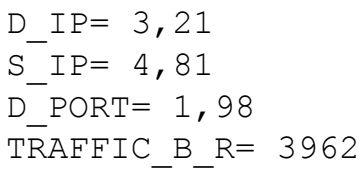

During next step MoA estimates the abnormality level of collected values. After this step MoA will be able to present its opinions about nodes states in a form of attack probabilities presented at the beginning of the section 5.

$\mathrm{MOA}_{1}\left(\mathrm{~N}_{1}, \mathrm{TCP}\right.$ FLAG $)=0,143$

$\mathrm{MOA}_{1}\left(\mathrm{~N}_{1}, \mathrm{D}_{-} \mathrm{IP}\right)=0,21$

$\mathrm{MOA}_{1}\left(\mathrm{~N}_{1}, \mathrm{~S}_{-} \mathrm{IP}\right)=0,81$

$\mathrm{MoA}_{1}\left(\mathrm{~N}_{1}, \mathrm{D}_{-} \mathrm{PORT}\right)=0,28$

where $\mathrm{N}_{1}$ - the node observed by $\mathrm{MoA}_{1}$

The probability values related to MoA observations are estimated with application of statistics presented in section 4. In general, the attack probability is greater, the current observation is more far from the historical records.

Third step performed by MoA is a comparison of current probability attack value with corresponding threshold value. For example:

$\mathrm{MOA}_{1}\left(\mathrm{~N}_{1}, \mathrm{TCP} \_\mathrm{FLAG}\right)<0,05$

$\mathrm{MOA}_{1}\left(\mathrm{~N}_{1}, \mathrm{D}_{-} \mathrm{IP}\right)<0,01$

$\mathrm{MoA}_{1}\left(\mathrm{~N}_{1}, \mathrm{~S}_{-}\right.$IP $)<0,5$

$\mathrm{MoA}_{1}\left(\mathrm{~N}_{1}, \mathrm{D}_{-} \mathrm{PORT}\right)<0,3$

As the result, MoA gets some binary vector:

\begin{tabular}{|c|c|c|c|c|}
\hline TRAFFIC_B_R & FLAG_SYN & D_IP & S_IP & D_PORT \\
\hline 0 & 0 & 0 & 0 & 1 \\
\hline
\end{tabular}

where ' 0 ' value in a vector means 'normal state' and ' 1 ' stands for "annomaly".

Next step is performed by a MA. The MA collects and processes binary vectors obtained from MoAs. The MA compares vectors to the known attack patterns.

For example the MA possesses the following list of MoA binary vectors:

$\operatorname{MOA}\left(\mathrm{N}_{1}, \mathrm{XXX}\right)$
\begin{tabular}{|c|c|c|c|c|}
\hline TRAFFIC_B_R & FLAG_SYN & D_IP & S_IP & D_PORT \\
\hline 1 & 1 & 0 & 0 & 0 \\
\hline
\end{tabular}

$\operatorname{MoA}\left(\mathrm{N}_{2}, \mathrm{XXX}\right)$

\begin{tabular}{|c|c|c|c|c|}
\hline TRAFFIC_B_R & FLAG_SYN & D_IP & S_IP & D_PORT \\
\hline 0 & 1 & 0 & 0 & 1 \\
\hline
\end{tabular}


$\operatorname{MoA}\left(\mathrm{N}_{3}, \mathrm{XXX}\right)$

\begin{tabular}{|c|c|c|c|c|}
\hline TRAFFIC_B_R & FLAG_SYN & D_IP & S_IP & D_PORT \\
\hline 0 & 1 & 1 & 1 & 1 \\
\hline
\end{tabular}

$\operatorname{MoA}\left(\mathrm{N}_{4}, \mathrm{XXX}\right)$

\begin{tabular}{|c|c|c|c|c|}
\hline TRAFFIC_B_R & FLAG_SYN & D_IP & S_IP & D_PORT \\
\hline 0 & 1 & 0 & 0 & 1 \\
\hline
\end{tabular}

$\operatorname{MOA}\left(\mathrm{N}_{5}, \mathrm{XXX}\right)$

\begin{tabular}{|c|c|c|c|c|}
\hline TRAFFIC_B_R & FLAG_SYN & D_IP & S_IP & D_PORT \\
\hline 0 & 0 & 0 & 0 & 1 \\
\hline
\end{tabular}

Comparing the binary vectors to attack patern defined in section $6 \mathrm{MA}$ recognizes the presense of reflector attack where node $\mathrm{N}_{3}$ plays a role of zombie node, node $\mathrm{N}_{4}$ and $\mathrm{N}_{2}$ play roles of reflectors and node $\mathrm{N}_{1}$ is a victim.

We consider only situation where exist exact mapping between MoA binary vectors and attack patern.

Second possible situation is that MA received a few different values of binary vectors from several independent MoAs that describe the same node $\mathrm{N}$ in the network. This unconsistency must be solved by MA otherwise it could not generate the final decision about the network security state. Simple voting or consensusbased conflict-solving methods are to be used here ${ }^{9}$.

Third scenario is that there may be several parallel attacks or abnormal situations in a corresponding node and this may also produce some not exact or inconsistent final results.

The reasoning about security events in theses three scenarios should also be considered and we plan to enhance our proposal with corresponding elements during further steps of our work.

\section{Conclusions}

In the paper we presented a new method for traffic anomalies detection based on Mark Burges statistics, observation of network traffic variables and the attack pattern ontology. As Mark Burgess technique has quite good ability to tolerate seasonal changes, do not require regularized data and requires relatively small set of data and utilizes CPU only on low level we hope that all this features will characterize also our proposal. These features are especially interesting in a context of real time identification performed on a single host and within mobile agent environments. Another important outcome of our work is the application of attack pattern ontology within a process of intrusion detection. The attack ontology allows us to efficiently combine observation coming from different sources (MoAs) and to draw final conclusion about current network security level. Additionally, a technique for observation of network communication patterns was proposed in order to reason about probability of unknown types of attacks and as an additional heuristic for tuning system parameters. All the above features were integrated within distributed multiagent infrastructure which is now under development and will be subject of further investigations.

\section{Acknowledgements}

This work was supported by the Polish State Committee for Scientific Research under Grant No. 3 T11C 02929 (2005-2007).

\section{References}

1. A. Beach, M. Modaff, Y.Chen, "Network Traffic Anomaly Detection and Characterization", cs.northwestern.edu/ ajb200/anomaly $\% 20$ detection $\% 20 p$ aper\%201.0.pdf.

2. M. Burgess, "An Approach to Understanding Policy Based on Autonomy and Voluntary Cooperation", DSOM 2005, pp. 97-108.

3. M. Burgess, "Two Dimensional Time-Series for Anomaly Detection and Regulation in Adaptive Systems", DSOM 2002, pp. 169-180.

4. V. Gorodetski, O. Karsaev, A. I. Khabalov, Kotenko, L. Popyack, V. Skormin, "Agentbased model of Computer Network Security System: A Case Study", In: Proceedings of International Workshop Mathematical Methods, Models and Architectures for Computer Network Security, Lecture Notes in Computer Science, vol. 2052, Springer Verlag, Berlin Heidelberg New York, 2001, pp. 39-50.

5. K. Hwang, H. Liu, Y. Chen, "Cooperative Anomaly and Intrusion Detection for Alert Correlation in Networked Computing Systems", Technical Report, USC Internet and Grid Computing Lab (TR 2004-16), 2004.

6. T.M. Khoshgoftaar, M.E. Abushadi, "Resource-sensitive intrusion detection models for network traffic", Eighth IEEE International Symposium on Publication, 2004, pp. 249- 258.

7. K Juszczyszyn, N.T. Nguyen, G. Kolaczek, A. Grzech, A. Pieczynska, R. Katarzyniak, "Agent-based Approach for Distributed Intrusion Detection System Design", International Conference on Computational Science 2006, United Kingdom, 2006, pp. 224-231.

8. K. Juszczyszyn, G. Kołaczek, "Assessing the Uncertainty of Communication Patterns in Distributed Intrusion 
Detection System", KES 2006, Lecture Notes in Artificial Intelligence 4252, 2006, pp. 243-250.

9. G. Kolaczek, A. Kuchtiak-Pieczynska, K. Juszczyszyn, A. Grzech, R. Katarzynak, N.T. Nguyen, "A Mobile Agent Approach to Intrusion Detection in Network Systems", Lecture Notes in Artificial Intelligence 3682, 2005, pp. 514-519.

10. I. Kotenko et al., "Multi-Agent Modeling and Simulation of Distributed Denial-of-Service Attacks on Computer Networks", In: Proceedings of Third International Conference Navy and Shipbuilding Nowaday. St. Petersburg, 2003, pp. 38-47.

11. M. Thottan, C. Ji., "Anomaly detection in IP networks", IEEE Transactions on Signal Processing, 51(8), 2003, pp. 2191- 2204.

12. A. Lakhina, M. Crovella, C. Diot, "Characterization of Network-Wide Anomalies in Traffic Flows", Technical Report BUCS-2004-020, Boston University, http://citeseer.ist.psu.edu/715839.html, 2004.

13. C.E. Shannon, W. Weaver, "The mathematical theory of communication", University of Illinois Press, Urbana, 1949.

14. N.Weaver, V. Paxson, S.Staniford, R. Cunningham, "A taxonomy of computer worms", ACM Workshop on Rapid Malcode - WORM '03, ACM Press, New York, NY, 2003, pp. 11-18.

15. Allmanz M. et.al. A First Look at Modern Enterprise Traffic, In Proc. Internet Measurement Conference, October 2005, pp. 217-231.

16. Faloutsos M., Faloutsos P., Faloutsos C., On power-law relationships of the Internet topology. In Proc.ACM SIGCOMM '99 Conference on Applications, Technologies, Architectures, and Protocols for Computer Communication, August 1999, pp. 251-262.

17. Kohler E., Liy J., Paxson V., Shenker S., Observed Structure of Addresses in IP Traffic, In Proc. SIGCOMM Internet Measurement Workshop, November 2002, pp. $253-266$.

18. Nicol D., Liljenstam M., Liu J., Multiscale Modeling and Simulation of Worm Effects on the Internet Routing Infrastructure, In Proc. Performance Tools Conference, 2003, pp. $1-10$.

19. G. Box, G. Jenkins, and G. Reinsel. Time Series Analysis. Prentice Hall, New Jersey, 1994.

20. M. Burgess. Two dimensional time-series for anomaly detection and regulation in adaptive systems. IFIP/IEEE 13th International Workshop on Distributed Systems: Operations and Management (DSOM 2002), 2002, pp. 169-181. 